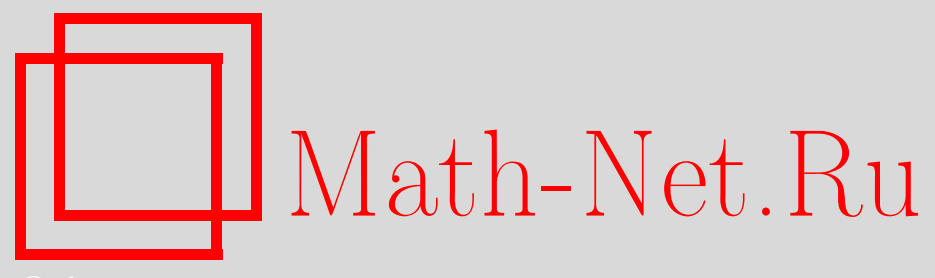

А. Рябченко, О распределении пар взаимно простых чисел, Квант, 2021, номер 5, 13-15

DOI: https://doi.org/10.4213/kvant20210502

Использование Общероссийского математического портала Math-Net.Ru подразумевает, что вы прочитали и согласны с пользовательским соглашением http://www . mathnet.ru/rus/agreement

Параметры загрузки:

IP : 54.198 .187 .58

26 апреля 2023 г., 12:54:43

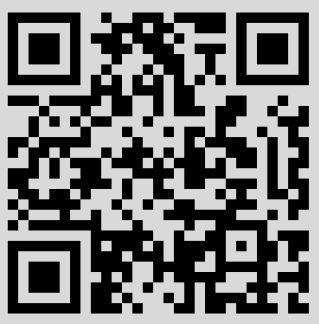




\section{О распределении пар взаимно простых чисел}

\section{А. РЯБЧЕНКО}

\section{Задача из XIX столетия}

В 1989 году в журнале «Квант» вышла статья Н.Я.Виленкина «В таинственном мире бесконечных рядов» [1]. В этой статье он рассказал о том, «как можно и как нельзя обращаться с бесконечными рядами». Один из наиболее известных рядов задает дзета-функцию Римана:

$$
\zeta(k)=\sum_{n=1}^{\infty} \frac{1}{n^{k}} .
$$

В качестве примера применения дзета-функции в статье Виленкина была разобрана известная вероятностная задача, о которой задумывались многие великие математики XIX века, такие как П.Дирихле, Л.Гегенбауэр, П.Л.Чебышёв и Э.Чезаро.

Задача. Какова вероятность того, что наудачу взятая дробъ несократима?

Обозначим через $f(N)$ количество несократимых дробей, числитель и знаменатель которых не превосходят $N$. В статье Виленкина показано, как найти $\lim _{N \rightarrow \infty} \frac{f(N)}{N^{2}}$. Это предел, к которому стремится доля несократимых дробей, когда правая граница отрезка $[1 ; N]$, из которого берутся числители и знаменатели, стремится к бесконечности. Оказывается, верно следующее:

$$
\begin{gathered}
\lim _{N \rightarrow \infty} \frac{f(N)}{N^{2}}=\left(1-\frac{1}{2^{2}}\right)\left(1-\frac{1}{3^{2}}\right)\left(1-\frac{1}{5^{2}}\right) \cdot \ldots= \\
=\prod_{i=1}^{\infty}\left(1-\frac{1}{p_{i}^{2}}\right)=\frac{1}{\sum_{n=1}^{\infty} \frac{1}{n^{2}}}=\frac{1}{\zeta(2)}=\frac{6}{\pi^{2}} .
\end{gathered}
$$

DOI: https://doi.org/10.4213/kvant20210502
Рассуждая нестрого, найти значение предела можно так. Так как и числитель, и знаменатель «взяты наудачу», вероятность того, что хотя бы один из них не делится на $i$-е простое число $p_{i}$, равна $1-\frac{1}{p_{i}^{2}}$. Если перемножить эти вероятности по всем простым числам, то после нескольких преобразований получится ответ $\frac{6}{\pi^{2}} \approx 0,60793$.

Для школьника, уже изучившего основную теорему арифметики, но еще не очень хорошо знакомого с понятием бесконечности в математике, такой ответ может показаться неубедительным. Ведь известно, что на каждую пару взаимно простых чисел $(a ; b)$ приходится бесконечное множество пар вида $(n \cdot a ; n \cdot b)$, где $n \geq 2$. А каждой паре $(n ; m)$ с общим делителем $d \geq 2$ соответствует единственная пара взаимно простых $(n / d ; m / d)$ с тем же отношением элементов. Исходя из этих фактов, должно было бы получиться, что для «по-настоящему случайных» пар чисел вероятность взаимной простоты (т.е. несократимости соответствующей дроби) равна нулю.

Однако так рассуждать по отношению к свойствам бесконечности неправильно. Приведенный выше ход мыслей использует несуществующую функцию равномерного распределения на множестве натуральных чисел. Иначе говоря, делается предположение, что взятое наудачу натуральное число может принимать любое, сколь угодно большое, значение с одинаковой вероятностью. Но тогда сумма вероятностей окажется равной $\infty$, а не 1 .

Иными словами, неясно, что именно означает фраза «наудачу взятая дробь» в 
условии задачи. Чтобы это понятие случайности было определено корректно, Виленкин в своей статье приводит решение задачи для конечного отрезка, а потом устремляет его длину к бесконечности. Для практических целей такой подход вполне допустим, ведь в очень многих ситуациях можно считать, что случайные числа берутся из очень большого, но тем не менее конечного отрезка.

\section{Функция Эйлера и случайные числа}

Итак, при стремлении длины отрезка $[1 ; N]$ к бесконечности вероятность того, что два случайных числа из него взаимно просты, стремится к $\frac{6}{\pi^{2}}$ (примерно 60,8\%). Это знание может помочь в обосновании некоторых пределов, содержащих известную функцию Эйлера.

Определение. Функция Эйлера $\varphi(n)-$ функция от натурального $n$, принимающая значение количества натуральных чисел, не превосходящих $n$ и взаимно простых с ним.

Верна следующая формула для $\varphi$ :

$$
\varphi\left(p_{\alpha_{1}}^{x_{1}} p_{\alpha_{2}}^{x_{2}} \cdot \ldots \cdot p_{\alpha_{k}}^{x_{k}}\right)=\prod_{i=1}^{k} p_{\alpha_{i}}^{x_{i}}\left(1-\frac{1}{p_{\alpha_{i}}}\right),
$$

где все $\alpha_{i}$ различны. Из этой формулы следует, что отношение $\varphi(n) / n$ бывает сколь угодно близким как к единице, так и к нулю. Действительно, для простого $n$ имеем $\varphi(n)=n-1$. Значит, при больших простых $n$ отношение $\varphi(n) / n$ близко к единице. С другой стороны, верен следующий факт: для любого $\varepsilon>0$ существует такое $m$, что выполнено неравенство $\prod_{i=1}^{m}\left(1-\frac{1}{p_{i}}\right)<\varepsilon$. Поэтому отношение $\varphi(n) / n$ может быть и сколь угодно малым. Это старый результат, но читатели могли видеть его упоминание в недавней статье В.Брагина [2]. Кроме того, в 1954 году польские математики А.Шинцель и В.Серпинский доказали (см. книгу Рибенбойма [3]), что множество значений, которые принимает функция $\varphi(n) / n$, плотно на $[0 ; 1]$.

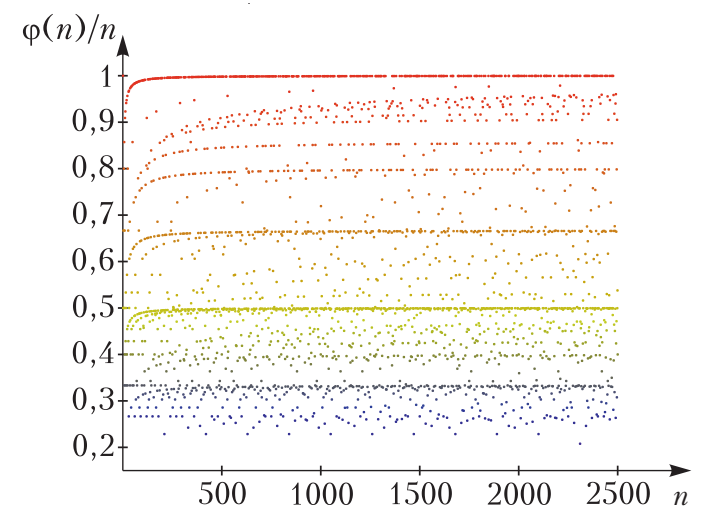

Рис. 1. Значения $\varphi(n) / n$ для $n$ от 1 до 2500

На графике $\varphi(n) / n$ (рис.1) можно выделить несколько покрытых одноцветными точками линий, асимптотами к которым являются прямые $y=1$ (красные), $y=1 / 2$ (желтые), $y=2 / 3$ (оранжевые) и т. д. Это явление объясняется тем, что большое простое число $p$ своим множителем $(1-1 / p)$ вносит маленький относительный вклад в значение $\varphi(n) / n$. Таким образом, произведения достаточно больших простых чисел, домноженные на 1 , будут давать значения $\varphi(n) / n$, близкие к 1 , домноженные на $2^{k}-$ к $1 / 2$, на $3^{k}-$ к $2 / 3$, на $2^{k} 3^{m}-$ к $1 / 3$ и т.д.

Несмотря на ясно наблюдаемую расходимость Значений функции $\varphi(n) / n$ при $n \rightarrow \infty$, для функции $g(N)=\sum_{n=1}^{N} \varphi(n)$ последовательность $\frac{g(N)}{N^{2}}$ имеет ненулевой предел.

Теорема. Выполняется соотношение $\lim _{N \rightarrow \infty} \frac{g(N)}{N^{2}}=\frac{1}{2} \lim _{N \rightarrow \infty} \frac{f(N)}{N^{2}}=\frac{3}{\pi^{2}} \approx 0,30396$.

Почему же $g(N)$ и $f(N)$ относятся в пропорции 1 к 2 при $N \rightarrow \infty$ ? Следующая визуализация позволяет эффектно ответить на этот вопрос.

Рассмотрим клетчатую плоскость, на которой отмечены все точки со взаимно простыми координатами (рис. 2). Ясно, что данная конструкция симметрична относительно прямой $x=y$. Кроме того, по определению верен тот факт, что на отрез- 


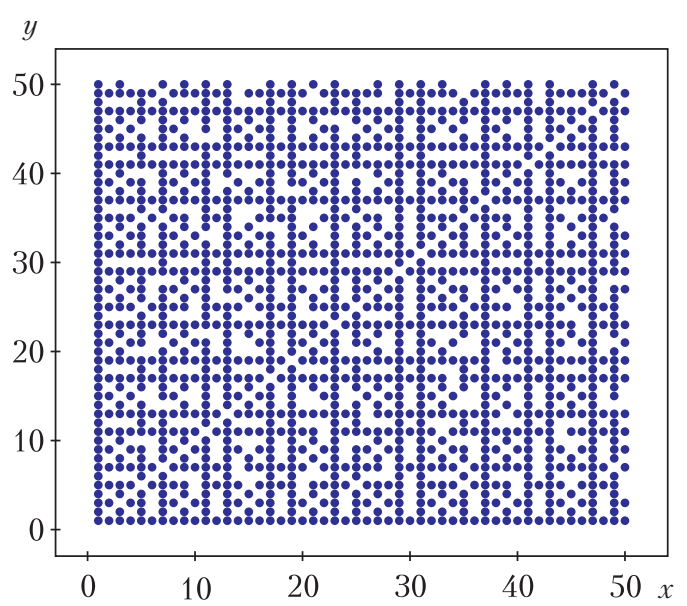

Puc. 2

ке, соединяющем точки $(n ; 1)$ и $(n ; n)$, выбрано ровно $\varphi(n)$ точек. Таким образом, в квадрате $[1 ; N] \times[1 ; N]$ ниже прямой $x=y$ лежит $\sum_{n=2}^{N} \varphi(n)=g(N)-1$ отмеченных точек, выше - столько же, и еще необходимо учесть точку $(1 ; 1)$.

Итого, в квадрате $[1 ; N] \times[1 ; N]$ лежит ровно $2 \cdot g(N)-1=2 \cdot \sum_{n=1}^{N} \varphi(n)-1$ точек, соответствующих несократимым дробям. Зная это, найти значение упомянутого ранее предела уже не представляет труда.

Доказательство теоремы. Искомый предел вычисляется следующим образом:

$$
\begin{array}{r}
\lim _{N \rightarrow \infty} \frac{g(N)}{N^{2}}=\lim _{N \rightarrow \infty} \frac{\sum_{n=1}^{N} \varphi(n)}{N^{2}}=\lim _{N \rightarrow \infty} \frac{1+f(N)}{2 N^{2}}= \\
=\frac{1}{2} \lim _{N \rightarrow \infty} \frac{f(N)}{N^{2}}=\frac{3}{\pi^{2}} .
\end{array}
$$

Теорема доказана.

Стоит упомянуть, что имеет место более сильное утверждение, доказательство которого значительно сложнее и требует некоторых знаний математического анализа (это доказательство изложено в книге [4]):

$$
\sum_{n=1}^{N} \varphi(n)=\frac{3}{\pi^{2}} N^{2}+O(N \log N) .
$$

Запись $O(N \log N)$ означает некоторую функцию от $h(N)$ такую, что $h(N)<$ $<C \cdot N \log N$ для некоторой константы $C$ при всех достаточно больших $N$.

\section{Задачи}

Здесь читатель найдет несколько задач по теме этой статьи, но в то же время не стоит думать, что все необходимое для их решения было упомянуто на предыдущих страницах. В любом случае, автор надеется, что тот, кто найдет в себе силы не только определить ответ, но и продумать полные решения этих задач, получит неподдельное эстетическое удовольствие.

Слова «случайный» и «взятый наудачу» в формулировках задач следует воспринимать как «взятый равновероятно из большого, но конечного отрезка», и задачи нужно решать, устремив его длину к бесконечности.

1. Найдите вероятность того, что взятое наудачу число не делится ни на один квадрат натурального числа, кроме единицы.

2. Найдите вероятность того, что наибольший общий делитель $k$ случайных чисел равен единице.

3. Придумайте ненулевую оценку снизу на вероятность того, что $k$ случайных чисел попарно взаимно просты ( $k$ фиксировано).

Подсказка. Для начала попробуйте найти вероятность того, что никакие два числа не делятся на фиксированное простое число $p$. Затем, воспользовавшись неравенством Бернулли, решите задачу.

4. Придумайте ненулевую оценку снизу на вероятность того, что произведение $k$ случайных чисел взаимно просто с произведением $l$ случайных чисел.

5. Найдите значение следующего предела:

$$
\lim _{N \rightarrow \infty} \frac{\sum_{n=1}^{N} \frac{\varphi(n)}{n}}{N} .
$$

Подсказка. Числитель и знаменатель из задачи про случайные дроби можно брать наудачу из отрезков разной длины. В некоторых случаях получится сохранить исходную плотность точек, соответствующих парам взаимно простых чисел.

\section{Литература}

1. Н.Виленкин. В таинственном мире бесконечных рядов. - «Квант», 1989, №10.

2. В.Брагин. Ряд чисел, обратных к простым. «Квант», 2019, №5.

3. P.Ribenboim. The New Book of Prime Number Records. - Springer, 1996.

4. G.H. Hardy, E.M.Wright. An Introduction to the Theory of Numbers ( $6^{\text {th }}$ edition). - Oxford University Press, 2008. 